\title{
Science, Measurement, and Technology Requirements for Infrared Climate Benchmark Missions
}

\author{
D.G. Johnson and M. Mlynczak \\ NASA Langley Research Center, Hampton, VA 23681 \\ David.G.Johnson@nasa.gov
}

\begin{abstract}
Quantifying climate change in the presence of natural variability requires highly accurate global measurements covering more than a decade. Instrument design considerations for trending terrestrial emitted radiance are described.

OCIS codes: (280.0280) Remote sensing and sensors; (280.4991) Passive remote sensing
\end{abstract}

\section{Introduction}

Quantifying climate change is challenging at a fundamental level. Consider the changes in global mean surface temperature observed over the past several decades, currently estimated to be on the order of $0.2 \mathrm{~K}$ per decade. The change that one might expect over the next decade is orders of magnitude smaller than diurnal and seasonal temperature cycles, and is smaller than interannual variability resulting from El Niño events. The magnitude of decadal climate change is also small compared to regional differences, and is comparable to the estimated measurement bias of the best sensors currently on orbit. Estimating long-term global changes in the presence of such large short-term regional variability requires an observation strategy carefully designed to reduce sampling bias below the expected level of decadal change.

Climate change studies typically consider trends in annual global means as a way to distinguish long-term change from short-term variability. Because so many individual observations are included in the mean, instrument precision is of less concern than instrument bias. Once precision is high enough to resolve natural variability with a signal-to-noise ratio (SNR) greater than two or three, the uncertainty in the mean will be limited by natural variability rather than instrument noise. There is also no need for spatial resolution to be high enough to distinguish clear and cloudy scenes, since all scenes are averaged together. As a result, the cost of climate change measurements can be controlled by trading precision, spatial resolution, and geographic fill factor for increased accuracy.

\section{Infrared Climate Benchmark Requirements}

A climate benchmark is a measurement that contains information about the current climate state and has sufficient accuracy to enable detection of climate change by comparison with future measurements. Because retrieval algorithms can introduce time-dependent biases of their own, benchmark measurements consist of direct observables. One such observable is radiance, although to be useful for studying climate change the radiance needs to be observed with sufficient spectral resolution and coverage to detect and distinguish changes in climate variables such as temperature, lapse rate, and humidity.
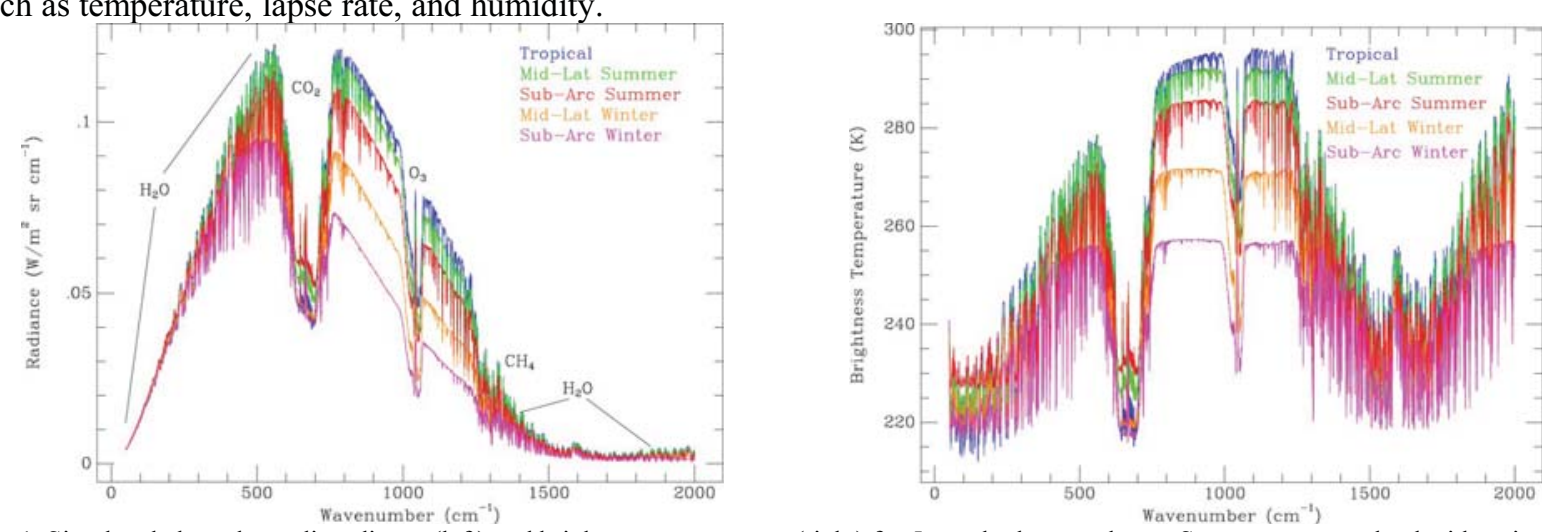

Fig. 1. Simulated clear-sky nadir radiance (left) and brightness temperature (right) for 5 standard atmospheres. Spectra are convolved with a sincVon Hann instrument line shape corresponding to an apodized resolution of $1.0 \mathrm{~cm}^{-1}$.

Thermal infrared spectra as shown in Figure 1 are a direct observable that satisfy the requirements for a climate benchmark. The region from $200-500 \mathrm{~cm}^{-1}$ contains information on the abundance and distribution of tropospheric water vapor. The shape of the $\mathrm{CO}_{2}$ band centered at $667 \mathrm{~cm}^{-1}$ depends on the temperature and pressure profile in the troposphere and stratosphere. Radiance in the window region between 800 and $1200 \mathrm{~cm}^{-1}$, on either side of the 
ozone band at $1000 \mathrm{~cm}^{-1}$, depends on surface temperature as well as cloud opacity and altitude. Finally, the integral over the full spectrum can be used to estimate the total emitted longwave flux for energy balance studies. Nominal requirements for an infrared benchmark measurement that maximizes information content and has sufficient accuracy for climate change studies [1] are summarized in Table 1.

Table 1: Infrared Benchmark Measurement Requirements

\begin{tabular}{|c|c|c|}
\hline Requirement & Value & Explanation \\
\hline Spectral range & $200-2000 \mathrm{~cm}^{-1}$ & $\begin{array}{l}\text { Covers most of thermal spectrum and provides sufficient information } \\
\text { to estimate the remainder with negligible uncertainty. }\end{array}$ \\
\hline $\begin{array}{l}\text { Spectral resolution } \\
\text { (unapodized) }\end{array}$ & $0.5 \mathrm{~cm}^{-1}$ & $\begin{array}{l}\text { Sufficient to resolve } 15 \mu \mathrm{m} \mathrm{CO} \mathrm{CO}_{2} \text { band and vertical temperature } \\
\text { structure }\end{array}$ \\
\hline Instrument bias (accuracy) & $0.1 \mathrm{~K}(\mathrm{k}=3)$ & $\begin{array}{l}\text { Accuracy sufficient that the ability to detect climate change is limited } \\
\text { by interannual variability and not instrument performance. }\end{array}$ \\
\hline Footprint & $25-100 \mathrm{~km}$ & $\begin{array}{l}\text { Footprint together with sample interval provides sufficient spatial } \\
\text { coverage to minimize sample bias. }\end{array}$ \\
\hline Ground sample interval & $200 \mathrm{~km}$ & Interval small enough to sample natural variability. \\
\hline
\end{tabular}

\section{Instrument Design and Performance Modeling}

To be useful as a climate benchmark the measurement accuracy needs to be known and verifiable on orbit. Achieving this requires that the instrument suite includes a verification system to evaluate known sources of measurement bias and to test for unexpected errors. A key component of this strategy is to include a radiance standard that can cover the expected range of scene radiance and verify the accuracy of corrections for error terms including nonlinearity, gain and offset drift, and polarization sensitivity [2].

The required optical throughput is modest since the spectrometer needs to obtain just a single calibrated nadir spectrum every $200 \mathrm{~km}$ along the ground track, corresponding to approximately one spectrum every $30 \mathrm{~s}$ at typical orbital altitudes. This allows the use of deep cavity blackbodies for calibration and for the verification source. The verification source also includes subsystems for measuring cavity emissivity and recalibrating the temperature sensors [3]. The relatively long interval between nadir observations makes it possible to include calibration blackbody and space views for each nadir observation, and to alternate nadir and verification system views. Frequent calibration views minimize the uncertainty resulting from gain and offset drift.

A Fourier transform spectrometer (FTS) is a good choice to meet the need for broad spectral coverage and high spectral resolution, given that no imaging is required. The performance modeling that follows is based on an FTS, although a similar analysis could be performed for a grating spectrometer. Currently available mature technology was selected for optics, detectors, beamsplitter/compensator pair, and the FTS carriage.

Detector and signal chain nonlinearity is a major concern in trying to meet the benchmark accuracy requirement. The effect of nonlinearity can be reduced in an FTS, although not eliminated entirely, by reducing the spectral band covered by a single detector. Three detectors were selected; a pyroelectric detector to cover $200-700 \mathrm{~cm}^{-1}$, a longwave (LW) $\mathrm{HgCdTe}$ detector to cover $600-1400 \mathrm{~cm}^{-1}$, and a midwave (MW) $\mathrm{HgCdTe}$ detector to cover 1000$2000 \mathrm{~cm}^{-1}$. The FTS is a 4-port design with two inputs and two outputs. One input views the scene, while the other views a stable internal reference source. One output illuminates the pyroelectric detector, while the other illuminates side-by-side LW and MW detectors. The input pupil is imaged inside the FTS, and reimaged onto the focal planes.

More than two dozen sources of instrument bias have been modeled and used as the basis for a set of instrument specifications. The set is not unique, but serves to illustrate some of the challenges of making a benchmark measurement. The resulting uncertainty rollup for the current configuration is shown in Figure 2. The midband uncertainty is limited by the calibration blackbody total temperature uncertainty specification of $17 \mathrm{mK}$. The rapid rise in uncertainty at the low frequency end of the pyroelectric band and the high frequency ends of the LW and MW $\mathrm{HgCdTe}$ bands is caused by nonlinearity, while the gradual increase in uncertainty with frequency for all bands (linear on the semilog plot, so the increase is exponential with wavenumber) results from the uncertainty in offset changes caused by thermal drift in the instrument, assuming that the instrument is near $273 \mathrm{~K}$. 


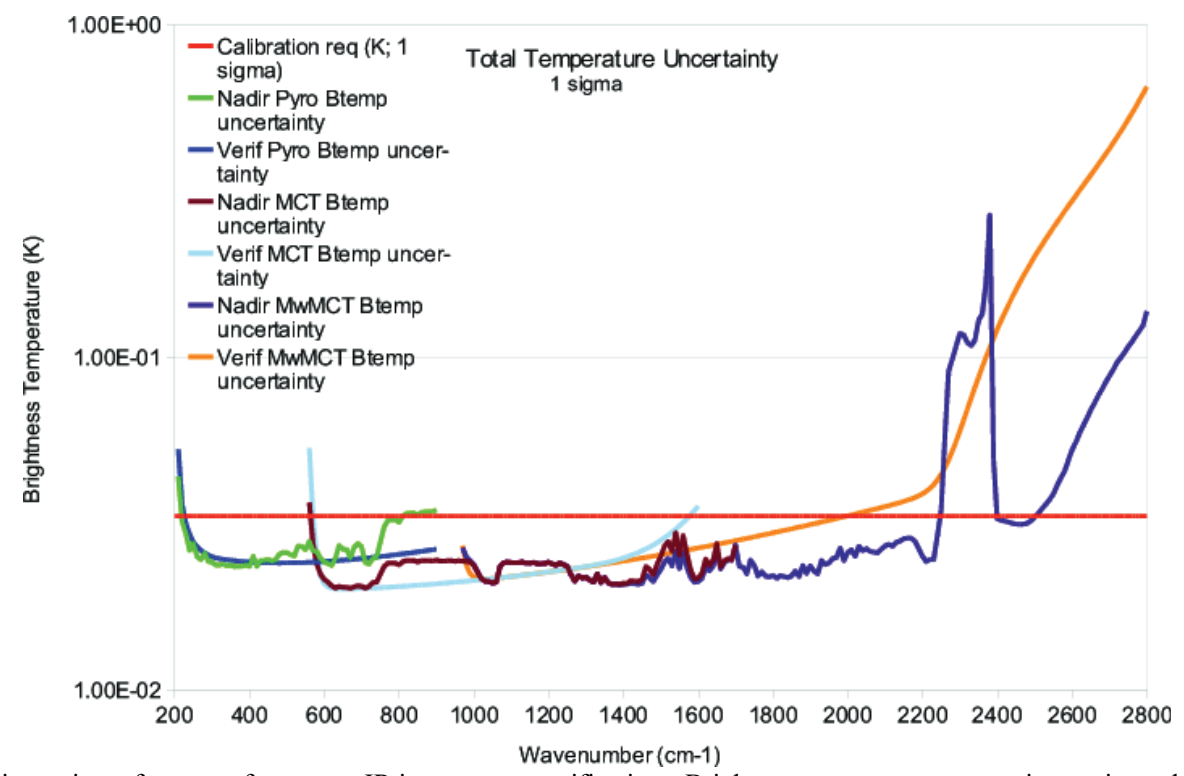

Fig. 2. Modeled radiometric performance for current IR instrument specifications. Brightness temperature uncertainty estimated for $233 \mathrm{~K}$ verification blackbody temperature and for nadir spectra calculated for midlatitude summer standard atmosphere. Accuracy estimated for pyroelectric (green, blue); LW HgCdTe (brown, cyan); and MW HgCdTe (purple, orange) channels. Requirement of $0.033 \mathrm{~K}$ ( $\mathrm{k}=1$, corresponding to $0.1 \mathrm{~K} \mathrm{k}=3$ ) shown in red.

\section{Summary}

It is possible to design an infrared spectrometer with sufficient accuracy to make climate benchmark measurements. Areas that require particular attention are detector and signal chain nonlinearities, thermal stability of all parts of the instrument that contribute to instrument background, and calibration source temperature measurement. A critical component of the instrument design is the ability to verify and fully characterize instrument performance on orbit.

\section{References}

[1] National Research Council, Earth Science and Applications from Space: Imperatives for the Next Decade and Beyond (National Academy Press, 2007), Chap. 4.

[2] J.A. Dykema and J.G. Anderson, "A methodology for obtaining on-orbit SI-traceable spectral radiance measurements in the thermal infrared," Metrologia 43, 287-293 (2006).

[3] F.A. Best, D.P. Adler, S.D. Ellington, D.J. Thielman, and H.E. Revercomb, "On-orbit absolute calibration of temperature with application to the CLARREO mission," in Earth Observing Systems XIII, J.J. Butler and J. Xiong, eds., Vol. 7081 of SPIE proceedings series (SPIE, Bellingham, Washington, 2008), doi:10.1117/12.795457. 